\title{
Schistosomiasis of Appendix with Review of Literature
}

\author{
Omaia Mahdy ${ }^{1}$, Nazima Haider ${ }^{2 *}$ and Sohaila Fatima ${ }^{2}$ \\ ${ }^{1}$ Department of Histopathology, Aseer central Hospital, Abha KSA \\ ${ }^{2}$ Department of Pathology, King Khalid University, Abha KSA
}

\begin{abstract}
Schistosomiasis a widespread parasitic disease is caused by a typical trematode with vertebrate-invertebrate lifecycle and occurs in welldefined geographical areas. The development of water resources and migration of populations are responsible for the introduction of the disease into new regions of the world. Schistosomiasis is an unusual cause of appendicitis. We present a case of appendicitis in a 60 year old Saudi male and discuss its review of literature.
\end{abstract}

Keywords: Schistosoma, Appendix

\section{Introduction}

Schistosomiasis is one of the most widespread yet neglected parasitic diseases in the world. They have a typical trematode vertebrate-invertebrate lifecycle, with humans being the definitive host. Environmental changes that result from the development of water resources

and migration of populations are responsible for the introduction of the disease into new geographic areas. Schistosomiasis of the appendix is a well-recognized disease but does not frequently cause appendicitis. Thus the condition is uncommon even in endemic regions. We hereby present a case of appendicitis in a 60 year old Saudi male to highlight this unusual cause of appendicitis and discuss its review of literature.

\section{Case report}

A 60 year old Saudi male presented with a history of on and off right iliac fossa pain for 2 years. He was seen by

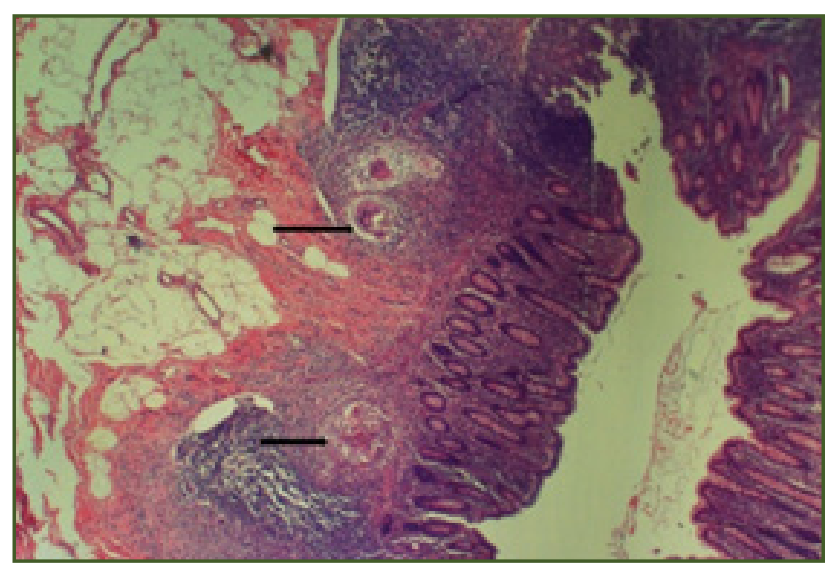

Fig. 1: Granulomatous inflammation (arrow) in appendix. Hematoxylin and eosin (10X). clinical practitioner who did an ultrasound which was unremarkable and he was treated with analgesics, antacids and anti spasmodic but there was no improvement. Besides the right iliac fossa pain, there were no other bowel or bladder complaints. On examination, the abdomen was soft but there was slight tenderness in the right lower quadrant on deep palpation. The rest of the abdominal examination was normal. The repeat ultrasound examination was also unremarkable. The full blood count was within normal range. A diagnosis of recurrent acute appendicitis was made and appendicectomy was performed. The appendix was slightly thickened and congested grossly. Histopathology of the appendix showed schistosoma eggs with lateral spine and granulomatous inflammation (Fig 1 \& 2) without any evidence of acute inflammation. Serum anti-bilharzias antibodies were positive but no schistosomal ova were found in urine and stool. He was given a single dose of Praziquantel $40 \mathrm{mg} / \mathrm{kg}$.

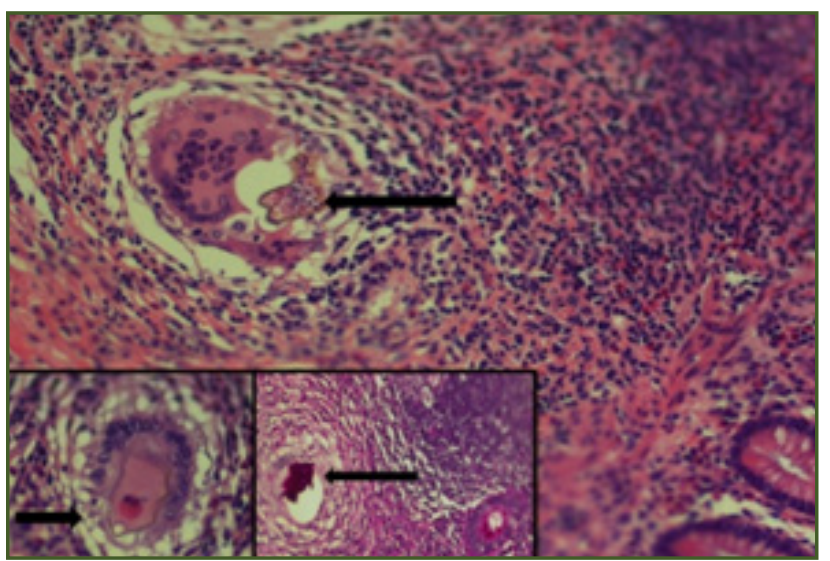

Fig. 2: Schistosoma eggs (arrow) with lateral spine. Hematoxylin and eosin (40X), Inset: Hematoxylin and eosin (40X), PAS (20X) 


\section{Discussion}

Schistosomiasis is a water-borne trematode infestation, affecting approximately 240 million people with 800 million are at risk. It occurs in well-defined geographical areas in tropics and subtropics particularly Africa, China and Middle East. [1]

Schistosomiasis was first described by Theodor Bilharz, hence also called bilharziasis , in 1851 but it was in 1908 when Pirajá da Silva first described the entire disease cycle. Cercariae, which escape from the snail (intermediate host) to the freshwater actively swim toward a human (definitive host) where they penetrate the skin directly, sheds tail, transform into a blood vessel inhabiting form termed the schistosomulum. These circulate with the blood as they develop to sexually mature blood flukes until they inhabit the portal hepatic tract (S. mansoni and S. japonicum) or the pelvic organs (S. haematobium). Eggs are released into the bloodstream and many embolize in smaller blood vessels and capillaries of diverse organs, inducing the characteristic granulomatous reaction of schistosomiasis. Passage of the eggs through the wall of bowel or bladder to the lumen from where they exit to the external environment in faeces or urine. [2]

Despite major advances in control and substantial decreases in morbidity and mortality, schistosomiasis continues to spread to new geographic areas. [2]

Two species S. mansoni and S. haematobium, are endemic in Saudi Arabia. Infection with S. mansoni was reported mainly in the highlands of the western areas and some parts of the Central and Northern Regions, whereas S. haematobium was reported mainly in lowland coastal plain in the southern areas. Biomphalaria pfeifferi and Bulinus beccarii are the species of fresh water snails incriminated in transmission of S. Mansoni and S. hematobium in Saudi Arabia, respectively. The hamadryas baboon (Papio hamadryas) is a species of baboon from the Old World monkey family. In Saudi Arabia, they inhabit an extensive range from the Yemen border in south to Akhal near Madinah in the northern Saudi Arabia. They use the same water sources in their habitat where human schistosomes have been recorded and proved to be natural reservoir hosts of many zoonotic diseases including S. mansoni infection. [3]

Schistosomiasis of the appendix was first described by Turner in 1909. [4] The incidence of schistosomal appendicitis is $0.02-6 \%$ in endemic areas but its very rare in developed countries. [5, 6]

Studies where schistosomiasis is endemic, have shown appendicitis to be relatively uncommon and the cause is not usually attributable to a schistosomal infestation of the appendix. The most common hypothesis is that eggs in the appendix wall stimulate inflammation and thus fibrosis and narrowing of the appendiceal lumen. Other hypotheses include schistosomal egg emboli causing ischaemia and granulomatous inflammation of the serosa and periappendicular tissue causing fibrosis. [6,7]

The patients may present with abdominal pain, vomiting and fever or altered bowel motion.

All the three species deposit eggs in the appendix. Gross examinations may show acute appendicitis with fibrinopurulent exudates, serosal congestion and thickened wall, fecaliths or it may be unremarkable. Confirmation of appendicular schistosomiasis is a purely histological diagnosis which shows the eggs, granulomatous inflammation with tissue eosinophilia and infiltrates of acute and chronic inflammatory cells. Morphologically, S. haematobium ova have a terminal spine while $S$. mansoni have lateral spine.[5,6,7] Some eggs are surrounded by a layer of eosinophilic material, the Splendore-Hoeppli phenomenon. [8]

Physicians must be aware of the possibility of seeing atypical presentations of parasitic diseases, especially considering the worldwide increase in immigration and tourism. Migrants, tourists and expatriates with chronic abdominal symptoms should also be asked about possible exposure. If an unexplained eosinophilia is detected, the respective parasitic and serologic tests should be performed. The detection of circulating antigens i.e. circulating cathodic antigen (CCA) and circulating anodic antigen (CAA) and schistosome DNA and RNA is proving to be a highly sensitive diagnostic approach. [9] Antihelmintic Praziquantel which acts on adult worms is in most common drug treating both urinary and gastrointestinal forms of the disease, and administered as a single dose. However, it's not effective against the immature, early schistosome stages. Artemisinin derivatives, such as artemether, is one such option which has the potential to kill the immature stages. $[10,11]$

\section{Conclusion}

Schistosomal appendicitis is an unusual presentation and histopathological examination is essential for its diagnosis. Such atypical presentations of parasitic diseases become more significant considering the worldwide increase in immigration and tourism.

\section{References}

1. WHO. 2014. Schistosomiasis fact sheet 115. World Health Organization, Geneva, Switzerland. 
2. Patz JA, Graczyk TK, Geller N, Vittor AY. Effects of environmental change on emerging parasitic diseases. International Journal for Parasitology. 2000;30:1395-405.

3. Lofty W M, Alsaqabi S M. Human schistosomiasis in the kingdom of Saudi Arabia: a review. Journal of the Medical Research Institute. 2010; 31: (1-6).

4. Turner SA. Bilharziasis of the appendix. Trans Mrd J. 1909; 5:210.

5. Badmos KB, Komolafe AO, Rotimi O. Schistosomiasis presenting as acute appendicitis. East Afr Med J. 2006. Oct;83(10):528-32.

6. Ahmed S A, Mohammed U, Sanda R B, Makama J, Shehu M S, Ameh E A, Mayun A A. Schistosomiasis of the Appendix in a Tertiary Hospital in Northern Nigeria: A 22-Year Review. Lab Physicians. 2014;6(1): 18-21. doi: 10.4103/0974-2727.129085.
7. Khan GM, Grillo IA, Abu-Eshy SA, Khan AR, Mubarak J, Jastaniah S. Pathology of appendix. J Natl Med Assoc. 2001;92: 533-5.

8. Edington GM, Gilles HM, editors. Pathology in the Tropics. London: Edward Arnold Publishers Ltd; 1976. pp. 149-79.

9. Weerakoon K G A D, Gobert G N, Cai P, McManus D P. Advances in the Diagnosis of Human Schistosomiasis. Clin. Microbiol. Rev. 2015 ; 28(4): 29 939-967. doi:10.1128/ CMR.00137-14

10. Bierman W F W, Wetsteyn J C F M, van Gool T. Presentation and diagnosis of imported schistosomiasis: relevance of eosinophilia, microscopy for ova, and serology. Journal of Travel Medicine. 2005; 12, (1): 9-13.

11. Liu Y, Wu W, Liang Y, Jie Z, Wang H, Wang W, Huang Y. New uses for old drugs: the tale of artemisinin derivatives in the elimination of schistosomiasis japonica in China. Molecules.2014. 19:15058-15074.

*Corresponding author:

Nazima Haider, Department of Pathology, King Khalid University, Abha KSA

Phone: +91 966-559103778

Email: nazima_haider@yahoo.com 